\title{
Investigation and Comparison between New Satellite Impact Test Results and NASA Standard Breakup Model
}

\author{
K. Sakuraba ${ }^{11}$, Y. Tsuruda ${ }^{1}$, T. Hanada ${ }^{1}$, J.-C. Liou ${ }^{2}$, Y. Akahoshi ${ }^{3}$ \\ ${ }^{1}$ Kyushu University, 744 Motooka, Nishi-ku, Fukuoka 819-0395, Japan \\ ${ }^{2}$ ESCG/ERC, Mail Code JE104, 2224 Bay Area Blvd., Houston, TX 77058, USA \\ ${ }^{3}$ Kyushu Institute of Technology, 1-1 Sensuicho, Tobata-ku, Kitakyushu 804-8550, Japan
}

\begin{abstract}
This paper summarizes two new satellite impact tests conducted in order to investigate on the outcome of low- and hyper-velocity impacts on two identical target satellites. The first experiment was performed at a low velocity of $1.5 \mathrm{~km} / \mathrm{s}$ using a 40 -gram aluminum alloy sphere, whereas the second experiment was performed at a hyper-velocity of $4.4 \mathrm{~km} / \mathrm{s}$ using a 4-gram aluminum alloy sphere by two-stage light gas gun in Kyushu Institute of Technology. To date, approximately 1,500 fragments from each impact test have been collected for detailed analysis. Each piece was analyzed based on the method used in the NASA Standard Breakup Model 2000 revision. The detailed analysis will conclude: 1 ) the similarity in mass distribution of fragments between lowand hyper-velocity impacts encourages the development of a general-purpose distribution model applicable for a wide impact velocity range, and 2) the difference in area-to-mass ratio distribution between the impact experiments and the NASA standard breakup model suggests to describe the area-to-mass ratio by a bi-normal distribution.
\end{abstract}

Keywords: Space Debris, Modelling, Impact Fragmentation.

\section{Introduction}

A commonly used model to describe the outcome of satellite fragmentation is the NASA Standard Breakup Model [1]. The model includes the size and area-to-mass distributions of fragments after an explosion or collision. It also includes the ejection velocity distribution of the fragments with respect to the parent object. The NASA model is an empirical model based on ground-based experiments and onorbit breakups. The database for collisions includes two non-catastrophic and three catastrophic impact experiments, and one on-orbit catastrophic collision, as summarized in Table 1 (see also Ref. [2]).

The outcome of a catastrophic collision is the total fragmentation of the target object, whereas a non-catastrophic collision only results in minor physical damage to the target. The transition from non-

\footnotetext{
${ }^{1}$ Corresponding author. Tel.: +81 90802 3048; fax: +81 928023001

E-mail address: mule-sakuraba@aero.kyushu-u.ac.jp
} 
catastrophic to catastrophic collisions appears to occur when the ratio of impact kinetic energy to target

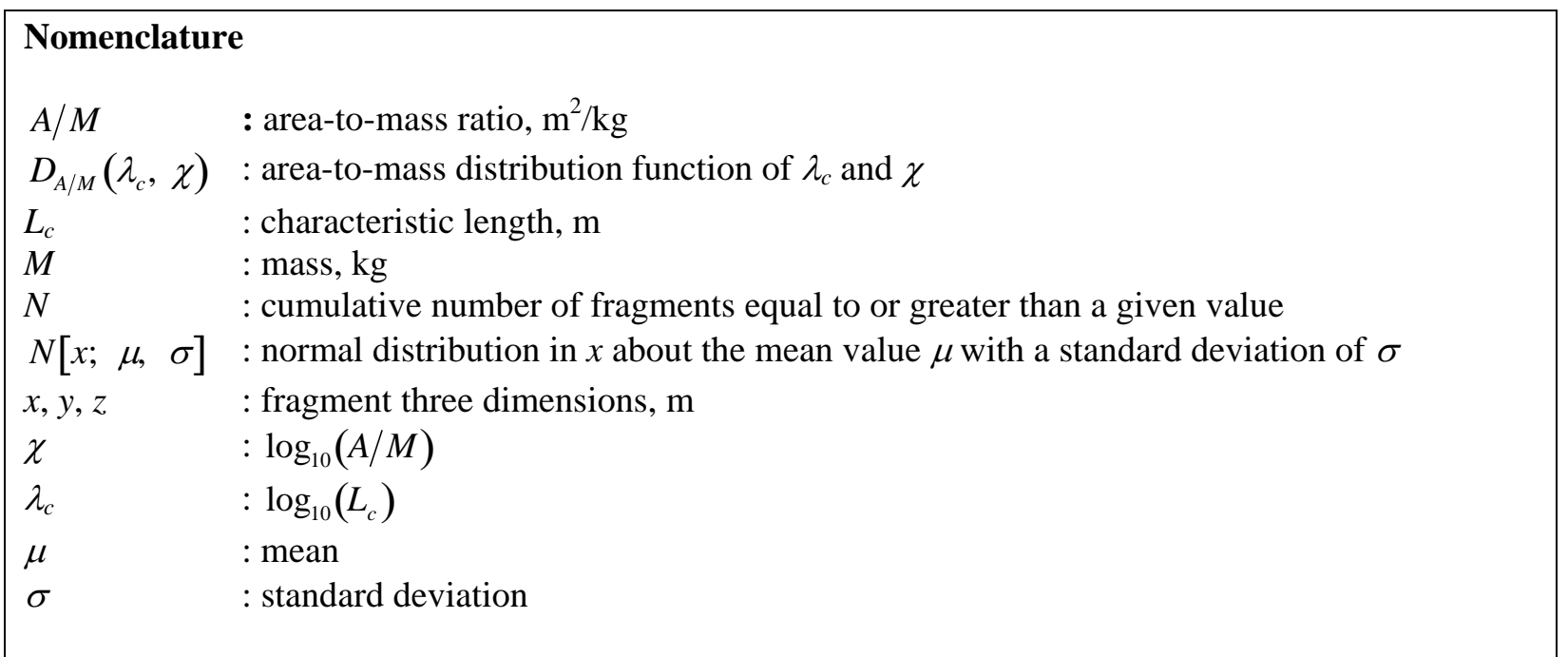

mass exceeds $40 \mathrm{~J} / \mathrm{g}$ [1]. As can be seen from Table 1, the experiments selected for the development of the NASA model were all in the hypervelocity impact regime, applicable to potential on-orbit collisions in the low-Earth orbit (LEO) region. A simple functional fit to the six fragment size distributions yield the following power-law equation,

$$
N_{\text {cum }}=0.1\left(M_{\text {tot }}\right)^{0.75}\left(L_{c}\right)^{-1.71}
$$

where $N_{\text {cum }}$ is the number of fragments larger than a given characteristic length, $L_{c}$ (in meters), and $M_{\text {tot }}$ is the total mass of the target and projectile (in kilograms). Overall, Eqn. (1) provides a reasonable fit to the six data sets, over more than twelve orders of magnitude in mass, or more than four orders of magnitude in size. For the area-to-mass ratio and ejection velocity distributions, the NASA collision model was derived based on additional on-orbit fragmentation data and the Satellite Orbital Debris Characterization Impact Test (SOCIT) data [1].

Yasaka et al. [3] investigated low-velocity impact phenomena, applicable for on-orbit collisions in the geosynchronous Earth orbit (GEO) region, while Johnson et al. [1] developed the NASA model described above. Yasaka et al. conducted experiments where a simulated spacecraft wall was impacted

Table 1. Comparison of impact experiments

\begin{tabular}{llllc}
\hline Name & Target Mass & Projectile Mass & Impact Velocity & Catastrophic? \\
\hline Bess 1 & - $^{(1)}$ & $1.65 \mathrm{~g}$ & $3.0 \mathrm{~km} / \mathrm{s}$ & No \\
Bess 2 & (1) $^{11}$ & $0.37 \mathrm{~g}$ & $4.5 \mathrm{~km} / \mathrm{s}$ & No \\
PSI 1 & $26 \mathrm{~kg}$ & $237 \mathrm{~g}$ & $5.9 \mathrm{~km} / \mathrm{s}$ & Yes \\
PSI 2 & $26 \mathrm{~kg}$ & $237 \mathrm{~g}$ & $3.3 \mathrm{~km} / \mathrm{s}$ & Yes \\
SOCIT & $34.5 \mathrm{~kg}$ & $150 \mathrm{~g}$ & $6.0 \mathrm{~km} / \mathrm{s}$ & Yes \\
P78/Solwind & $850 \mathrm{~kg}$ & $16 \mathrm{~kg}$ & $7.6 \mathrm{~km} / \mathrm{s}$ & Yes \\
\hline
\end{tabular}

(1) The target was a simulated spacecraft wall. 


\section{K. Sakuraba / International Journal of Impact Engineering}

by solid stainless steel spheres at a speed of $300 \mathrm{~m} / \mathrm{s}$ or lower to develop a low-velocity collision model in a similar manner to what had been done in the area of hypervelocity impacts. The cumulative size distribution of fragments was expressed in mass by the following power-law equation,

$$
N_{\text {cum }}=0.78\left(M_{f} / M_{e}\right)^{-0.68}
$$

where $N_{\text {cum }}$ is the number of fragments weight more than a given mass, $M_{f}$ (in grams), and $M_{e}$ is the total mass of fragments (in grams). Yasaka et al. also provided the ejection velocity distribution of the fragments with respect to the parent object based on an empirical equation derived by Su [4] and McKnight [5] from a variety of on-orbit fragmentations. Many international groups working on orbital debris evolutionary models for the GEO region have adopted their low-velocity collision model as well. Hanada et al. [6] reanalyzed the low-velocity impact data obtained by Yasaka et al. [3] based on the analytic method used in the NASA model, and then compared the results with the NASA model. It was concluded that the NASA model could be applied to low-velocity collisions with some simple modifications. Their conclusion has encouraged us to develop a wide velocity range collision model. The low-velocity impact experiments conducted by Yasaka et al. [3] were categorized as noncatastrophic collisions, characterized primarily by fragmentation of the projectile and by crater or hole on the target, however.

The purpose of this paper is to investigate the outcome of low- and hyper-velocity catastrophic impacts on two identical target satellites. One was hit with a 40-gram aluminum alloy sphere at a lowvelocity of $1.5 \mathrm{~km} / \mathrm{s}$ whereas the other one was hit with a 4-gram aluminum alloy sphere at a hypervelocity of $4.4 \mathrm{~km} / \mathrm{s}$. The ratios of impact energy to target mass for the two experiments were approximately the same $(55 \mathrm{~J} / \mathrm{g})$. The target satellites were completely fragmented in both experiments, consistent with the NASA criterion mentioned previously. Approximately 1,500 fragments from each impact experiment have been weighed, measured, and analyzed based on the analytic method used in the NASA standard breakup model 2000 revision.

\section{NASA Standard Breakup Model}

The NASA standard breakup model 2000 revision [1] is quite different from other fragmentation models. Previously, mass and diameter (or size) were used interchangeably as the independent variable. However, with the incorporation of area-to-mass ratio distributions, this interchangeability is lost, and then characteristic length $L_{c}$ is chosen as an independent variable. The following subsections will describe the hypervelocity collision model adopted in the NASA standard breakup model 2000 revision.

\subsection{Size Distribution}

Collisions between two satellites may be non-catastrophic, characterized primarily by fragmentation of the smaller object and by cratering of the larger object, or catastrophic, wherein both objects are totally fragmented. The difference between a catastrophic and a non-catastrophic collision is determined by the ratio of kinetic energy at impact to target mass. If the ratio is equal to or greater than $40 \mathrm{~J} / \mathrm{g}$, then the collision is catastrophic. Based on several laboratory hypervelocity impact experiments, including the highly instrumented Satellite Orbital Debris Characterization Impact Test (SOCIT) series as well as the on-orbit collision of the Solwind spacecraft, the number of fragments of a 
given size and larger can be described by Eqn. (1) mentioned previously.

\subsection{Area-to-Mass Ratio}

For objects with $L_{c}$ smaller than $8 \mathrm{~cm}$, a single area-to-mass ratio $A / M$ distribution function has derived from hypervelocity impact experiments as follows:

$$
D_{A / M}^{S O C}\left(\lambda_{c}, \chi\right)=N\left[\chi ; \mu^{S O C}\left(\lambda_{c}\right), \sigma^{S O C}\left(\lambda_{c}\right)\right]
$$

where

$$
\lambda_{c}=\log _{10} L_{c}, \chi=\log _{10} A / M
$$

and $N$ is a normal distribution in $\chi$ about the mean value of

$$
\mu^{S O C}\left(\lambda_{c}\right)=\left\{\begin{array}{cc}
-0.3 & \lambda_{c} \leq-1.75 \\
-0.3-1.4\left(\lambda_{c}+1.75\right)-1.75 & \leq \lambda_{c}<-1.25 \\
-1.0 & \lambda_{c} \geq-1.25
\end{array}\right.
$$

with a standard deviation of

$$
\sigma^{S O C}\left(\lambda_{c}\right)=\left\{\begin{array}{cc}
0.2 & \lambda_{c} \leq-3.5 \\
0.2+0.1333\left(\lambda_{c}+3.5\right) & \lambda_{c}>-3.5
\end{array}\right.
$$

Note that the $A / M$ distribution function given by Eqn. (3) is assumed to describe adequately the $A / M$ characteristic of small debris produced in the explosive breakup of either spacecraft or rocket booster.

\section{Impact Experiment}

\subsection{Two-Stage Light Gas Gun}

Figure 1 shows the two-stage light gas gun at Kyushu Institute of Technology, Japan. It consists of ignition chamber, pump tube, launch tube, and vacuum chamber. At first, the piston beside the ignition chamber is accelerated by ignition power in ignition chamber toward the coupling section to compress light gas in the pump tube. At the second stage, the diaphragm between the free flight section and the launch tube is ruptured by the compressed light gas and then the gas starts to accelerate the projectile.

\subsection{Target Satellite}

The targets for the two impact experiments were identical micro satellites, $150 \times 150 \times 150 \mathrm{~mm}$ in size with a communication antenna on the top and solar cells on a side as shown in Table 2 . The main structure of each micro satellite was composed of five layers (top, bottom, and internal three layers 


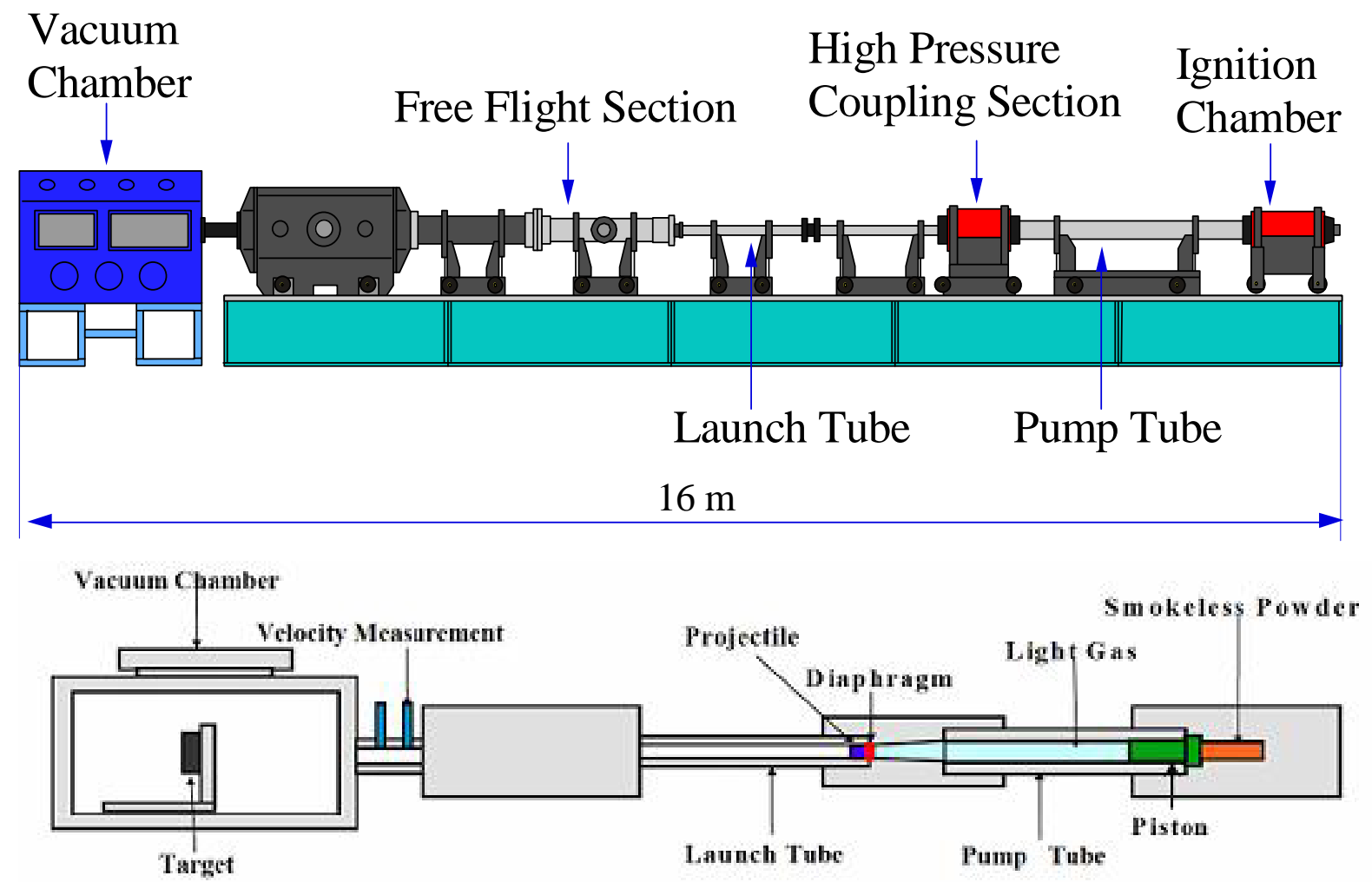

Fig. 1. Two-stage light gas gun.

parallel to the top and bottom layers) and four side panels. Unlike the target prepared for the previous impact experiment that was a cylindrical-shaped micro satellite without side panels [7], this new structure made the target more realistic. The external layers and side panels were made of Carbon Fiber Reinforced Plastic (CFRP), the internal three layers were made of Glass Fiber Reinforced Plastic (GFRP), assembled by angle bars made of an aluminum alloy and plastic spacers. The thickness of the external layers was $2 \mathrm{~mm}$, while that of the internal layers and the four side panels was $1 \mathrm{~mm}$. The interior of each micro satellite was equipped with fully functional wireless radios, lithium-ion batteries, communication circuit, electric power supply circuit, and command and data handling circuit. The total mass of each micro satellite was 740 grams. A special fragment recovery box was designed to hold the target satellite (see Fig. 2).

Two different solid spheres, made of an aluminum alloy, were prepared as projectiles. One was 30 $\mathrm{mm}$ in diameter and 40 grams in mass, whereas the other was $14 \mathrm{~mm}$ in diameter and 4 grams in mass. The projectiles were launched from the two-stage light gas gun at Kyushu Institute of Technology described above. The first experiment was performed at low-velocity of $1.5 \mathrm{~km} / \mathrm{s}$ using the 40 -gram aluminum alloy sphere. The second experiment was performed at hypervelocity of $4.4 \mathrm{~km} / \mathrm{s}$ using the 14-gram aluminum alloy sphere. The ratios of impact kinetic energy to target mass for the two experiments were approximately the same $(55 \mathrm{~J} / \mathrm{g})$, and placed the outcome as catastrophic according to 
the NASA criterion mentioned previously.

Table 2. Configuration of target satellite.

\begin{tabular}{ll}
\hline Target & CubeSat (cubed micro satellite) \\
Dimension $[\mathrm{mm}]$ & $150 \times 150 \times 150$ \\
Mass $[\mathrm{g}]$ & 740 \\
Density $\left[\mathrm{kg} / \mathrm{m}^{3}\right]$ & 219 \\
Materials & CFRP, \\
& $\begin{array}{l}\text { GFRP, } \\
\text { Aluminum alloys, and } \\
\text { plastics } \\
\text { wireless radio, } \\
\text { lithium-ion batteries, } \\
\text { components } \\
\text { electric power supply circuit, } \\
\text { data and command handling circuit, and } \\
\text { body mounted solar cells }\end{array}$ \\
Overview &
\end{tabular}

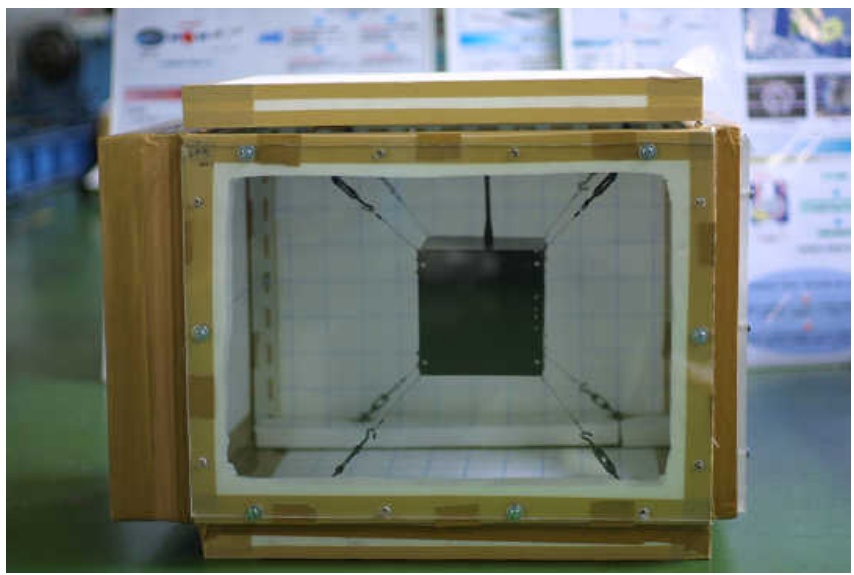




\section{K. Sakuraba / International Journal of Impact Engineering}

Fig. 2. Fragments recovery box.

\section{Experiment Results and Discussions}

\subsection{Overview of Impact Experiment Results}

Figure 3 shows fragmentation of the target satellite right after the impact experiment. Both target satellites were completely fragmented after the impact, consistent with the NASA criterion mentioned previously. The projectile of the low-velocity impact was partially fragmented while the projectile of the hyper-velocity impact was completely fragmented beyond recognition.

Figure 4 compares the 500 largest fragments from each impact experiment. The CFRP side panel and layer fragments are easily recognizable among the pieces. The overall characteristics of the two fragment sets are similar, although some differences exist. For example, many line-shaped fragments were generated by the hypervelocity impact (compare fragments at the lower right in Fig. 4), whereas only a few line-shaped fragments were generated by the low-velocity impact [8]. Such a difference may lead to differences in fragment properties as described later.
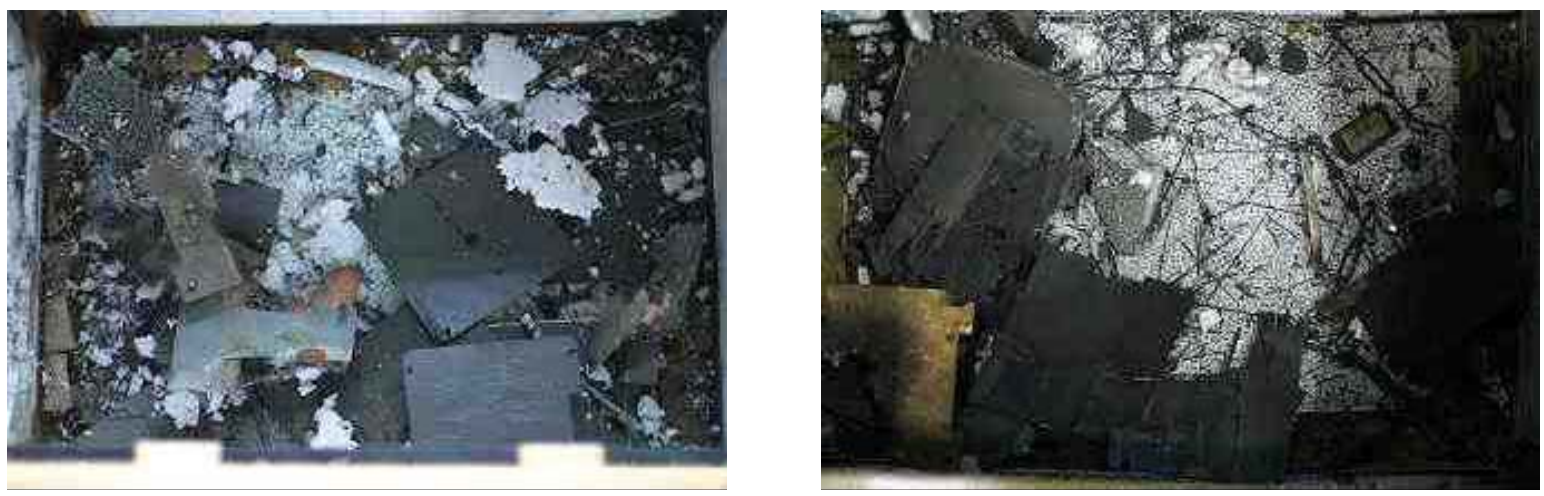

Fig. 3. Fragmentation of the target satellites: (left) low-velocity impact; (right) hypervelocity impact. 

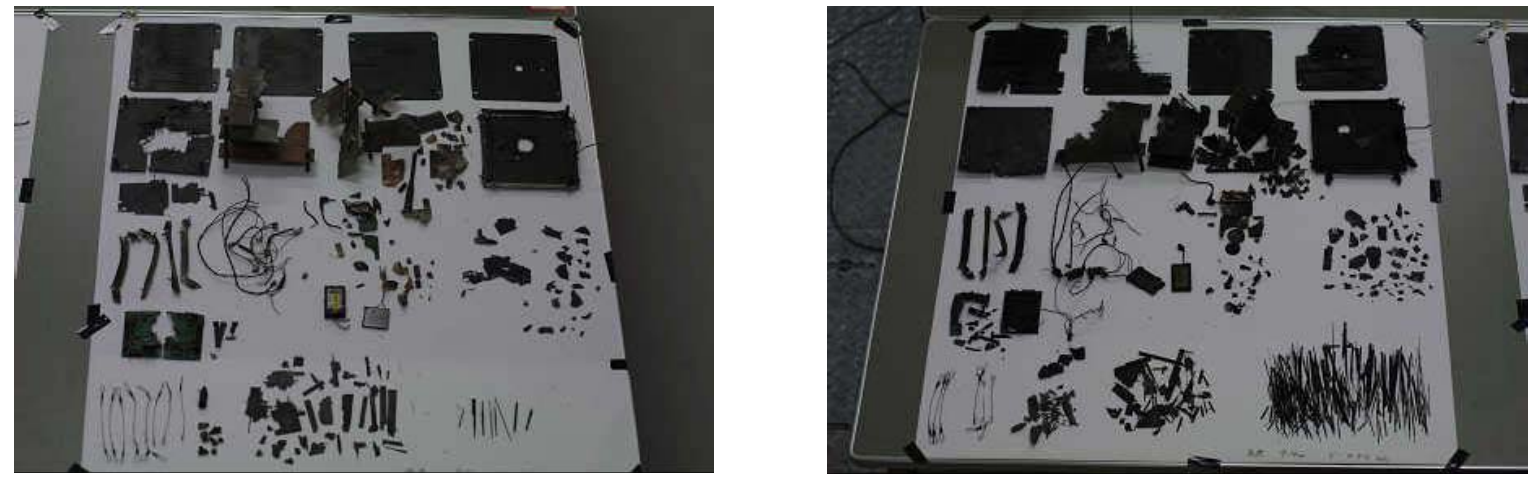

Fig. 4. Five hundreds largest fragments: (left) low-velocity impact; (right) hypervelocity impact.

\subsection{Size and Mass Distributions}

To date, approximately 1,500 fragments from each impact experiment have been collected for detailed analysis. Each piece has been weighed and measured based on the analytic method used in the NASA standard breakup model. These fragments account for more than $90 \%$ of the target mass in both impact experiments.

The cumulative distribution of collision fragments can be expressed in characteristic length or in mass. The characteristic length of an object is defined as the average of three orthogonal dimensions, $x$, $y$, and $z$, where $x$ is the longest dimension, $y$ is the longest dimension in the plane perpendicular to $x$, and $z$ is the longest dimension perpendicular to both $x$ and $y$. Figure 5 shows size distribution, i.e. number of fragments equal to or greater than a given size. It can be observed from Fig. 5 that a leveling off of the data occurs at the smaller size range. This is caused by the difficulty in collecting and measuring fragments smaller than several millimeters. Figure 5 also compares the size distributions of the two fragment groups with those produced by Eqn. (1), i.e. the NASA model. Even though there are some noticeable difference between the two fragment groups, Eqn. (1) provides a reasonable fit to the data sets. For better fitting, however, it could be recommended for Eqn. (1) to be shift slightly toward the larger size range.

Figure 6 shows mass distribution, i.e. number of fragments equal to or greater than a given mass. As shown in Fig. 6, the two fragment groups are similar in mass distribution. This similarity in mass distribution encourages the development of a general-purpose mass-based distribution model applicable for a wide impact velocity range.

\subsection{Area-to-Mass Ratio Distribution}

Figure 7 shows the area-to-mass ratio distribution of the two fragment groups with those produced by the NASA model. As given in Eqn. (3), the NASA model expresses the area-to-mass ratio distribution as a single normal distribution in $\chi$ and its mean value and standard deviation are a function of $\lambda_{c}$. To make the comparison easier, therefore, the area-to-mass ratio distribution of the two fragment groups was split into several bins based on the value of $\lambda_{c}$ at an interval of 0.2. As shown in Fig. 7, the 


\section{K. Sakuraba / International Journal of Impact Engineering}

area-to-mass ratio distribution of the two fragment groups show two peaks, high and low area-to-mass ratio groups, corresponding to low- and high-density material groups. Since the NASA standard breakup model was based on impact experiments using satellite made of high-density materials the NASA model fits well to the first peak, i.e. the area-to-mass ratio distribution of the high-density material group. The target satellites impacted were made of modern materials like CFRP and GFRP. To incorporate the area-to-mass ratio distribution of the low-density material group, it could be recommended to express it by a bi-normal distribution. For better fitting, however, we have to investigate the fraction between low- and high-density material groups.

\section{Conclusion}

This paper summarized two new satellite impact experiments conducted to investigate the outcome of low- and hyper-velocity impacts on two identical satellites. One was hit with a 40-gram aluminum alloy sphere at a low-velocity of $1.5 \mathrm{~km} / \mathrm{s}$, whereas the other was hit with a 4-gram aluminum alloy

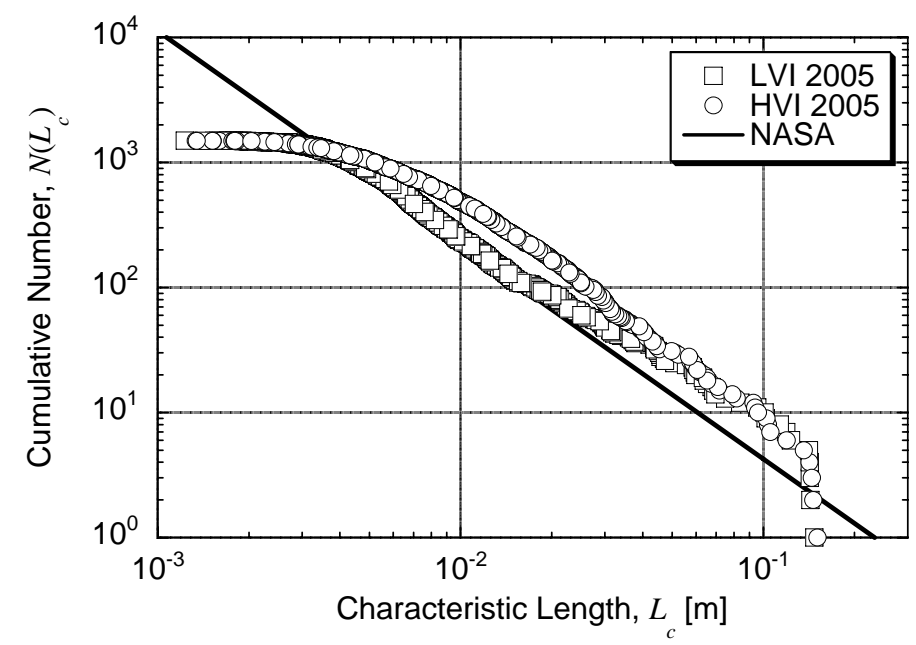

Fig. 5. Comparison of size distribution between experimental results and NASA standard breakup model. 
K. Sakuraba / International Journal of Impact Engineering

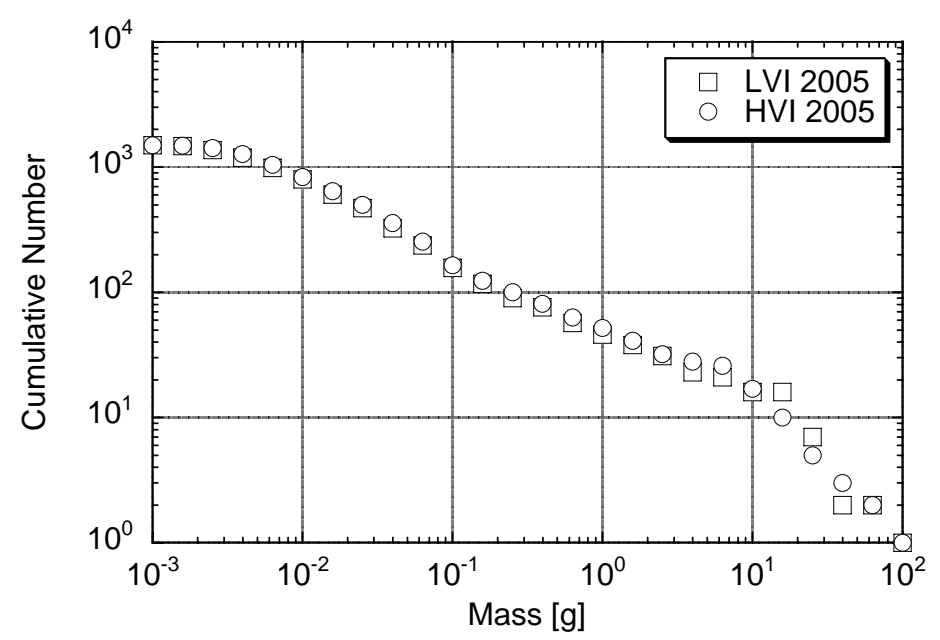

Fig. 6. Mass distributions of the two fragment sets.

sphere at a hyper-velocity of $4.4 \mathrm{~km} / \mathrm{s}$. The target satellites were completely fragmented in both impact experiments. Approximately 1,500 fragments from each impact experiment have been collected and analyzed based on the method used in the NASA standard breakup model. The results presented in this paper have indicated the following conclusions:

1. The similarity in mass distribution of fragments between low- and hyper-velocity impacts encourages the development of a general-purpose mass-based distribution model applicable for a wide impact velocity range.
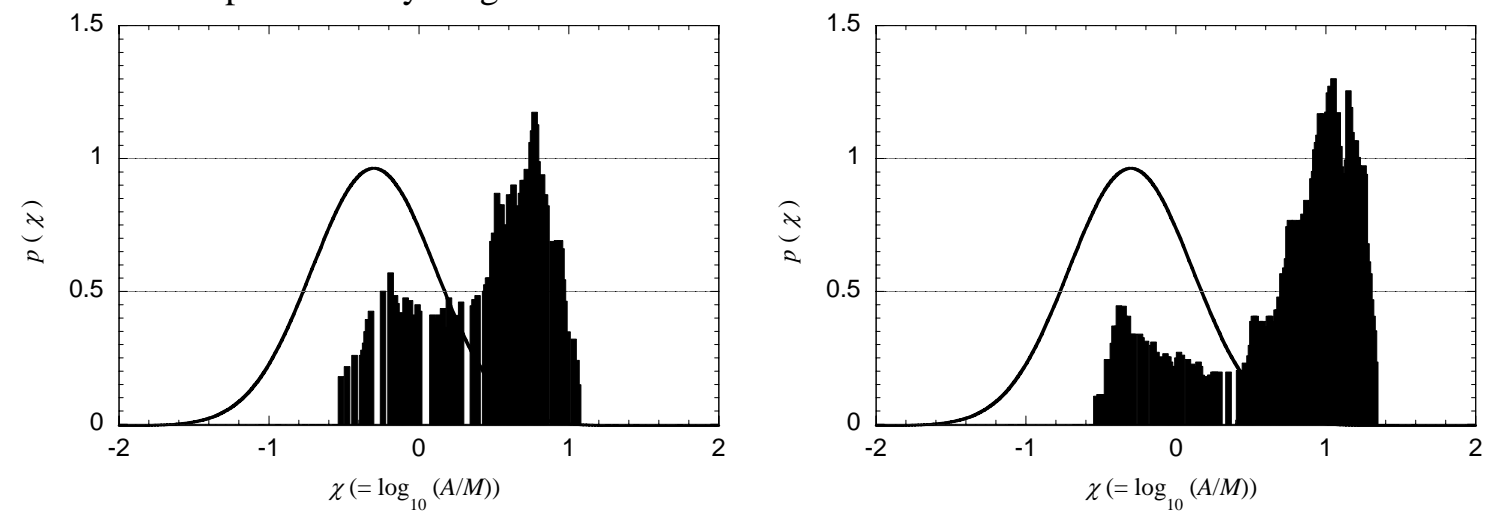

(a) $-1.8<\lambda_{\mathrm{c}}<-2.0$; NASA model with $\lambda_{c}=-1.9$. 

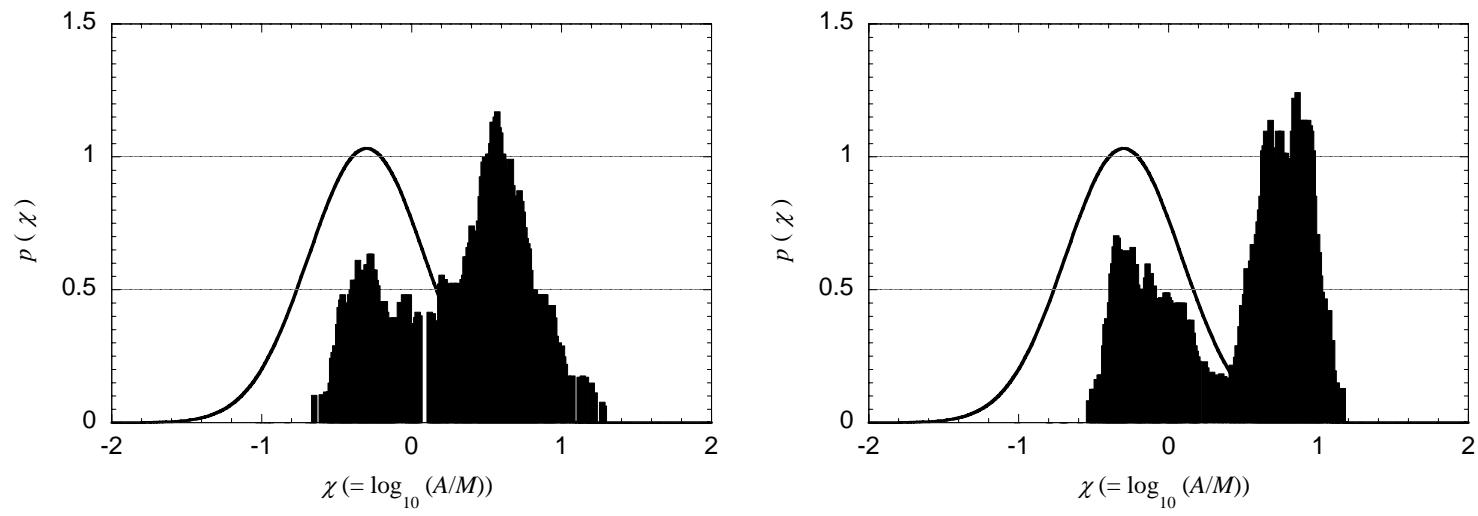

(b) $-2.0<\lambda_{\mathrm{c}}<-2.2$; NASA model with $\lambda_{c}=-2.1$.
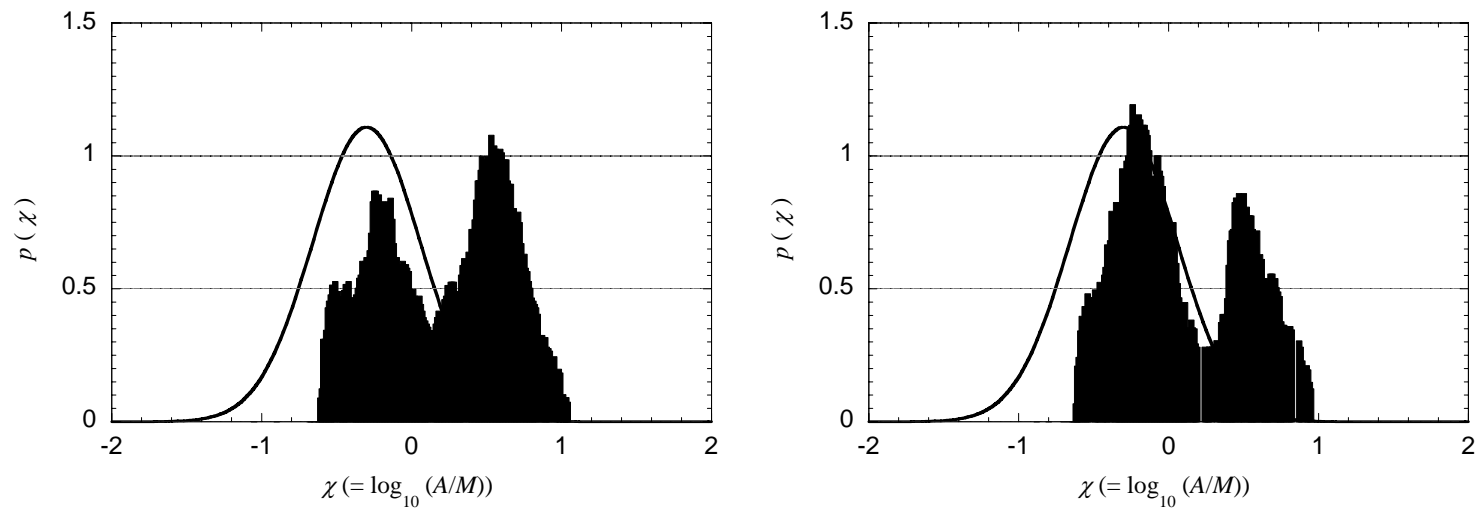

(c) $-2.2<\lambda_{\mathrm{c}}<-2.4$; NASA model with $\lambda_{c}=-2.3$.

Fig. 7. Comparison of probability density distribution of area-to-mass ratio: (left) low-velocity impact; (right) hypervelocity impact.

2. The difference in the area-to-mass ratio distribution between the impact experiments and the NASA model suggests describing the area-to-mass ration by a bi-normal distribution (not a single normal distribution as the NASA standard breakup model does).

\section{Acknowledgment}

The authors acknowledge the generous support of the NASA Orbital Debris Program Office on the experiments. The authors also acknowledge the practical advice of Professor Takashi Nakajima of JAXA Institute of Space and Astronautical Science on the test results. 


\section{Reference}

[1] Johnson, N. L., Krisko, P. H., Liou, J.-C. et al. NASA’s New Breakup Model of EVOLVE 4.0. Adv. Space Res., 2001; 28(9): 1377-1384.

[2] Liou, J.-C., Johnson, N. L., Krisko, P. H. et al. The New NASA Orbital Debris Breakup Model. In: Green, S.F. et al.. editors, Dust in the Solar System and Other Planetary Systems, Pergamon, London, UK, 2002. p.363-367.

[3] Yasaka, T., Hanada, T., Hirayama, H. Low-velocity Projectile Impact on Spacecraft. Acta Astronautica, 2000; 47(10): 763-770.

[4] Su, S.-Y. The Velocity Distribution of Collisional Fragments and Its Effect on Future Space Debris Environment. Adv. Space Res., 1990; 10(3-4): 389-392.

[5] McKnight, D. S. Determination of Breakup Initial Conditions. J. Spacecraft, 1991; 28(4): 470-477.

[6] Nakashima, K., Hanada, T., Akahoshi, Y. et al. Low-velocity Catastrophic Impact on Micro Satellite. Proc. 4th European Conference on Space Debris, Darmstadt, Germany, 2005: p.701-704.

[7] Hanada, T., Yasaka, T., Hata, H. et al. Using NASA Standard Breakup Model to Describe Low-Velocity Impacts on Spacecraft. J. Spacecraft, 2005; 42(5): 859-864.

[8] Hanada, T. and Liou, J.-C. Comparison of Fragments Created by Low- and Hyper-velocity Impacts. Adv. Space Res. 2007 (in press, doi:10.1016/j.asr.2007.05.062). 